\title{
Creating a virtual community of practice: an evaluation of ophthalmology-optometry Project ECHO
}

\author{
Michael Williams $\mathbb{D}^{1} \cdot$ Augusto Azuara Blanco ${ }^{2,3} \cdot{\text { Ruth } \mathrm{Hogg}^{2}}^{2}$ Gerry Mahon ${ }^{3} \cdot$ Margaret McMullan $^{4}$. \\ Raymond Curran ${ }^{4} \cdot$ Max Watson ${ }^{5}$
}

Received: 3 July 2018 / Accepted: 6 July 2018 / Published online: 1 August 2018

(c) The Royal College of Ophthalmologists 2018

As the absolute number of elderly people rises and with the advent of OCT scanners, community optometrists (COs) are seeing more abnormalities in their routine practice. Furthermore hospital eye services face a challenge keeping up with demand. Project ECHO (Extension for Community Healthcare Outcomes) is an educational model that partners primary healthcare providers with specialists, creating a 'community of practice'. ECHO has been applied to many areas of healthcare (http://echo. unm.edu accessed June 2018), but not previously to eye care. We describe the first ECHO project involving ophthalmologists (in the 'hub') and COs (in the 'spokes'). Some COs want enhanced roles: ECHO is a potential vehicle contributing to governance of shared eye care. The aims of this study were to assess the feasibility, acceptability and impact on knowledge and self-reported efficacy of the eye care ECHO and explore participants' impressions of it.

A mixed-methods prospective cohort study was performed on 12 ECHO sessions, held weekly. Each session had the same $21 \mathrm{COs}$, and in the hub one of two ophthalmologists who focused on macular disease or glaucoma. A talk was given by a hub-member, then COs presented cases. Due to the small sample size, descriptive statistics

Michael Williams

m.williams@qub.ac.uk

1 Centre for Medical Education, Queen's University of Belfast, Belfast, UK

2 Centre for Public Health, Queen's University of Belfast, Belfast, UK

3 Belfast Health and Social Care Trust, Belfast, UK

4 Northern Ireland Health and Social Care Board, Belfast, UK

5 Northern Ireland Hospice, Belfast, UK and non-parametric tests were used. Focus group data was analysed using thematic analysis.

Of the 21 spoke optometrists, $29 \%(6 / 21)$ had OCT scanners. In a knowledge assessment, marks improved from the pre-ECHO test (median 25/38, range 19-31), to the post-ECHO test (median 27/38, range 21-33) $(z=-2.3$, $p=0.019)$. Ninety-one percent (10/11) of COs who responded indicated they had learnt "a lot" through ECHO and 70\% (7/10) agreed that participating in ECHO had improved the care they provide for patients "a lot". Analysis of transcribed focus group discussions led to identification of three themes: professional and clinical learning occurring, expectations being exceeded, and suggestions for future ECHO sessions. An exemplar quotation was: “...certainly for me it will reduce some unnecessary referrals but it will also help me pick up on things that I really need to refer". Also 'diffusion of learning' occurred: "I found other colleagues asking me things and it was something that I could maybe bring as a question in ECHO, so the whole team did learn, and because I work in different practices, that was spread among different practices too."

Though numbers are small, the data suggest that ECHO was feasible, acceptable and could be an effective training modality. Focus groups discussions by participants in the ECHoES trial (unrelated to Project ECHO) revealed potential barriers to the deployment of optometrists in hospital-based ophthalmic care, including the need for "buy in" for optometric involvement [1]. 'Buy in' was suggested to depend on training delivered in a way that would reassure the ophthalmologist: ECHO may provide this.

As the ECHO model is replicable, the challenge would be to nurture local relationships formed to ensure sustainable participation, and eventually to incorporate ECHO's multidisciplinary networks permanently into service delivery in a way that is acceptable to all, safe and demonstrably benefits patients. 
Acknowledgements

Funding This ECHO project was supported by funding from the Northern Ireland Health and Social Care Board.

\section{Compliance with ethical standards}

Conflict of interest The authors declare that they have no conflict of interest.

\section{Reference}

1. Reeves BC, Scott LJ, Taylor J, Hogg R, Rogers CA, Wordsworth S, et al. The Effectiveness, cost-effectiveness and acceptability of Community versus Hospital Eye Service followup for patients with neovascular age-related macular degeneration with quiescent disease (ECHoES): a virtual randomised balanced incomplete block trial. Health Technol Assess. 2016;20: $1-120$.

\title{
Post-injection endophthalmitis in eyes receiving vs. not receiving topical antibiotic prophylaxis in Northern Thailand
}

\author{
Voraporn Chaikitmongkol $^{1} \cdot$ Onnisa Nanegrungsunk ${ }^{2} \cdot$ Direk Patikulsila $^{1} \cdot$ Janejit Choovuthayakorn ${ }^{1}$. \\ Nawat Watanachai ${ }^{1} \cdot$ Paradee Kunavisarut $^{1} \cdot$ Nimitr Ittipunkul $^{1} \cdot$ Neil M. Bressler $^{3}$
}

Received: 8 February 2018 / Revised: 26 April 2018 / Accepted: 7 May 2018 / Published online: 14 August 2018

(c) The Royal College of Ophthalmologists 2018

\section{Introduction}

Endophthalmitis following intravitreous anti-vascular endothelial growth factor (VEGF) injection is a rare sight-threatening condition. Several Western references suggest lower rates of post-injection endophthalmitis can be achieved without topical antibiotic prophylaxis [1-3]. However, a majority of physicians in the Asia-Pacific region still prescribe antibiotic prophylaxis [4], anecdotally stating concern that differences in environmental factors (e.g., tropical climates) or patient factors in the region might cause higher risk of endophthalmitis, comparing to the Western settings, to warrant this prophylaxis, despite little scientific rationale supporting such use. To our knowledge, little is known regarding incidence of post-injection endophthalmitis without antibiotic

Neil M. Bressler

nmboffice@jhmi.edu

1 Retina Division, Department of Ophthalmology, Faculty of Medicine, Chiang Mai University, Chiang Mai, Thailand

2 Department of Ophthalmology, Faculty of Medicine, Chiang Mai University, Chiang Mai, Thailand

3 Retina Division, Wilmer Eye Institute, Johns Hopkins University School of Medicine, Baltimore, MD, USA prophylaxis in a developing countries in Asia or elsewhere. Therefore, this study determined incidence of postinjection endophthalmitis with vs. without topical antibiotic prophylaxis at a university-based practice in Northern Thailand.

\section{Materials and methods}

IRB-approved prospective case series of patients receiving anti-VEGF injections at Chiang Mai University Hospital between May 2015 and September 2016 with follow-up anticipated for $\geq 3$ weeks after injections were recruited. Before injections, study eyes were examined by slit-lamp biomicroscopy, ensuring no intraocular inflammation. Endophthalmitis was evaluated at $4 \pm 1$ weeks after the injection, and defined as severe inflammation in both anterior chamber and vitreous cavity associated with pain, redness, or decreased vision, regardless of whether subsequent cultures were positive.

A standardized intravitreous anti-VEGF injection protocol was strictly applied, including use of sterile drape, sterile eyelid speculum, sterile glove, procedure mask, and application of povidone-iodine, twice, over eyelid and conjunctival sac, and over injection site using a povidone-iodine soaked cotton tip for $\geq 30 \mathrm{~s}$ before injection. 